\title{
APPLICATION OF ARTIFICIAL NEURAL NETWORKS FOR PREDICTING TENSILE INDEX AND BRIGHTNESS IN BLEACHING PULP
}

\author{
Onur Tolga Okan ${ }^{\text {I,^, Ithan Deniz }}{ }^{1}$, Sebahattin Tiryaki ${ }^{I}$
}

\begin{abstract}
The purpose of this study was to develop artificial neural network (ANN) models for predicting the effects of wood species, sodium perborate tetrahydrate (SPBTH) ratio, time, and beating degree on tensile index and brightness in bleaching pulp. Unbleached kraft-AQ bamboo and poplar pulps were exposed to first stage oxygen delignification for bleaching under $0,5 \mathrm{MPa}, 3 \% \mathrm{NaOH}$ and $12 \%$ consistency conditions. SPBTH bleaching was then carried out as the final stage. SPBTH bleached pulp was next beaten using two different degrees (55 $\mathrm{SR}^{\circ}$ and $65 \mathrm{SR}^{\circ}$ ). Tensile index and brightness data for training, validation and testing of the models were elicited from these experimental investigations. The models were established using the resulting data. The lowest $\mathrm{R}^{2}$ value was 0,98 among training, testing and validation data sets in the prediction of both tensile index and brightness. The networks therefore explain at least $98 \%$ of the experimental data for all data sets. The results indicate that ANN is a useful and effective tool for predicting tensile index and brightness. This study thus describes a novel and alternative approach to predicting tensile index and brightness in bleaching pulp compared to the literature.
\end{abstract}

Keywords: Bleaching process, neural network modeling, Phyllostachys bambusoides, Populus euramericana, pulping properties.

\section{INTRODUCTION}

The main problems facing the pulp and paper industry are inadequate supplies of raw materials and the effect of contamination by bleaching chemical reagent in recent decades (Okan et al. 2013). In order to resolve the problem of inadequate raw materials, there is growing emphasis on the use of alternative raw materials, particularly non-wood waste and fast-growing plants (Ferrer et al. 2011). Bamboo and poplar, which are fast-growing plants, are widely used as a raw material for the production of paper and paperboard across the world (Atchinson 1998, Xu et al. 2010). Another important problem facing the paper industry concerns minimizing environmental pollution. These problems have led the pulp industry to develop new technologies which do not cause environmental problems in the production of bleached paper. Environmentally friendly bleaching procedures have thus replaced the classical steps based on chlorine and or chlorinated compounds with others that use alternative reagents such as hydrogen peroxide, oxygen and ozone (Jimenez et al. 1999, Atik et al. 2006, Moldes et al. 2008, Okan et al. 2013).

New developments and markets have increased the demands on pulp and paper, and thus on pulp quality. The visual appearance and strength of the package is crucial for attracting customers (Nykvist 2006). Brightness and tensile index are therefore among the most important quality parameters of commercial pulps. The aim of bleaching is to obtain high and stable brightness at a desired level while minimizing chemical and bleaching costs. The physical and chemical composition of the raw material and the desired characteristic (brightness and strength) of the finished paper are also very important in selecting bleaching technologies (Rajesh and Ray 2006).

\footnotetext{
${ }^{1}$ Karadeniz Technical University, Faculty of Forestry, Department of Forest Industry Engineering, 61080 Trabzon, Turkey.

^ Corresponding author: onurtolgaokan@ktu.edu.tr

Received: 07.07.2014 Accepted: 15.12.2014
} 
Some previous studies have investigated the effects of the sodium perborate ratio, bleaching time and beating degree on brightness and tensile index. Varennes et al. (1996), investigated the effects of the sodium perborate ratio (2-6,5\%) and bleaching time (0-120 $\mathrm{min})$ on brightness of unbleached thermomechanical pulp. They observed that brightness values rose as the sodium perborate ratio and bleaching time were increased. Sain et al. (1997), reported a negative effect of a long bleaching time at high temperature but a positive effect of the sodium perborate ratio on pulp brightness. Peşman et al. (2014), investigated optical properties of deinked pulps exposed to sodium perborate at $0,25 \%$ to $1 \%$. They then determined that brightness value increased along with increases in the sodium perborate ratio. Lopez et al. (2002), determined optimum conditions in brightness and tensile index values in the sodium perborate bleaching applied to olive tree residue pulp at a short sodium perborate consistency (5\%) and medium to short bleaching time (60-120 min).

Experimental studies have proved that many process parameters affect the tensile index and brightness in bleaching pulp. Investigating the effect of each parameter on tensile index and brightness is expensive, tiring and time-consuming. Using more economical methods to obtain the desired results is important for solving these problems. Artificial neural networks (ANNs) have been reported to be commonly used for this purpose in various fields of application (Esteban et al. 2011). However, only limited information is available for predicting the effect of the various process parameters on tensile index and brightness in bleaching pulp using ANN models. Zainuddin et al. (2011), predicted tensile index values with an accuracy level of 96,47\% in the oil palm frond pulping process using an ANN model.

The main objective of this study was to predict tensile index and brightness using an ANN model for the purpose of obtaining more economical and reliable results without performing comprehensive tests in the pulp bleaching process.

\section{Artificial neural networks (ANNs)}

ANNs are one of the most attractive branches of artificial intelligence and have been used in many engineering applications for prediction, optimization, classification and data processing (Canakci et al. 2012, Tiryaki and Hamzacebi 2014). The technique has been developed as a powerful modeling method compared to statistical or quantitative methods (Ceylan 2008). The most widely used type of ANN for predictive purposes is Multi-Layer Perceptron (MLP). A typical example of the MLP structure is given in figure 1 (Hamzacebi 2011).

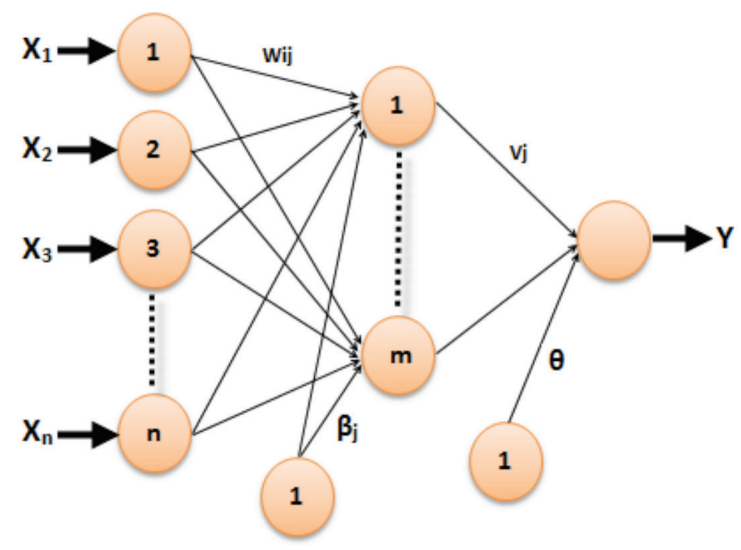

Figure 1. A typical MLP structure. 
Equation 1 gives the mathematical expression of the output of the MLP shown in figure 1.

$$
\mathrm{Y}=\mathrm{g}\left(\theta+\sum_{\mathrm{j}=1}^{\mathrm{m}} \mathrm{v}_{\mathrm{j}}\left[\sum_{\mathrm{i}=1}^{\mathrm{n}} \mathrm{f}\left(\mathrm{w}_{\mathrm{ij}} \mathrm{X}_{\mathrm{i}}+\beta_{\mathrm{j}}\right)\right]\right)
$$

where $\mathrm{Y}$ is the prediction value of a dependent variable, $\mathrm{X}_{\mathrm{i}}$ is the input value of the $\mathrm{i}^{\text {th }}$ independent variable, $\mathrm{w}_{\mathrm{ij}}$ is the weight of connection between the $\mathrm{i}^{\text {th }}$ input neuron and the $j^{\text {th }}$ hidden neuron, $\beta_{\mathrm{j}}$ is the bias value of the $j^{\text {th }}$ hidden neuron, $v_{j}$ is the connection weight between the $j^{\text {th }}$ hidden neuron and output neuron, $\theta$ is the bias value of the output neuron and $g($.$) and f($.$) are the activation functions of output and hidden neurons, respectively.$

MLP is composed of an input layer which takes data from outside, an output layer which exudes the results of the network, and usually one but occasionally more than one hidden layer between the input and output layers. The number of input neurons, hidden neurons and output neurons in the layers and activation function significantly influence the performance of the established models. The number of input neurons is equal to the number of independent variables, and the number of output neurons is equal to the number of dependent variables in a prediction problem based on a cause and effect relationship. Heuristic approaches are generally used to determine the number of hidden neurons (Baily and Thompson 1990, Lippmann 1987, Masters 1993, Hamzacebi et al. 2009).

\section{MATERIALS AND METHODS}

\section{Materials}

Bamboo (Phyllostachys bambusoides) and poplar (Populus euramericana (Dode) Guinier) were chosen as the experiment materials. The bamboo was obtained from Rize/Pazar and poplar from Trabzon, both in Turkey. The bamboo samples were 5-7 cm in diameter, with nodes and rotten parts removed from the trunk. Bamboo was manually chopped to $30 \times 20 \times 4 \mathrm{~mm}$. The bamboo chips were screened, air dried and stored at a dry solid content of $92 \%$. Poplar diameter was $20-25 \mathrm{~cm}$, with knots and rotten parts again removed from the trunk. Poplar was manually chopped to $1,5-2 \times 20-25 \mathrm{~mm}$ for pulping and was screened, air dried and stored at a dry solid content of $90 \%$.

\section{Chemicals}

Sodium hydroxide pellets $(99,9 \%)$, antraquinone $(97 \%)$ and ethylenediaminetetraacetic (EDTA) were obtained from Sigma-Aldrich. Magnesium sulfate and SPBTH were obtained from Merck. Oxygen gases used in the experiments were purchased from commercial sources.

\section{Pulping Procedure}

In order to ensure that pulp kappa numbers were within the category of easily and moderately bleachable pulps, previous studies were referenced for bamboo and poplar (Francis et al. 2006, Rahmati et al. 2010). The pulp from bamboo and poplar chips in this study was produced by the Kraft-AQ method. Table 1 shows the cooking conditions and pulp properties. 
Table 1. Cooking conditions and properties of bamboo and poplar wood chips.

\begin{tabular}{lll}
\hline Conditions & Bamboo & Poplar \\
\hline E.A. (as NaOH) \% & 18 & 16 \\
Sulfidity (\%) & 25 & 25 \\
AQ ratio (\%) & 0,1 & 0,1 \\
Liquor to wood ratio $(1 / \mathrm{kg})$ & $4 / 1$ & $5 / 1$ \\
Cooking Temperature $\left({ }^{\circ} \mathrm{C}\right)$ & 165 & 165 \\
Time (min.) & 90 & 120 \\
Viscosity $\left(\mathrm{cm}^{3} / \mathrm{g}\right)$ & 1164 & 1168 \\
Kappa no & 20,03 & 22,40 \\
Screened Yield $(\%)$ & 45,8 & 59,56 \\
Reject $(\%)$ & 0,25 & 0,21 \\
Total Yield $(\%)$ & 46,05 & 59,77 \\
\hline
\end{tabular}

\section{Bleaching Procedure}

A totally chlorine-free bleaching sequence consisting of an oxygen stage (O), a chelating stage (E) and a SPBTH stage was carried out with bamboo and poplar pulps. Oxygen delignification was performed in a thermostatically controlled, electrically heated, rotary, laboratory-type reactor with a capacity of 15 liters, resistant to pressure of $25 \mathrm{~kg} / \mathrm{m}^{2}, 4 \mathrm{rpm}$, with an automated temperature control table. The reactor was manually loaded and unloaded, and $600 \mathrm{~g}$ of fully dry chips were used for all types of cooking procedure. Six different bleaching experiments were performed in order to determine optimum oxygen delignification conditions for bamboo and poplar pulp (Table 2). A consistency of $12 \%$, sodium hydroxide concentration of $3 \%$ and magnesium sulphate concentration of $0,5 \%$ were used in all instances. Reaction temperature and reaction time were constant $100{ }^{\circ} \mathrm{C}$ and $60 \mathrm{~min}$, respectively.

Table 2. Optimization properties of oxygen delignification on bamboo and poplar pulps.

\begin{tabular}{cccccc}
\hline Wood species & $\begin{array}{c}\mathrm{O}_{2} \\
\text { Pres. }\end{array}$ & Kappa No & $\begin{array}{c}\text { Pulp } \\
\text { Viscosity } \\
\left(\mathrm{cm}^{3} / \mathrm{g}\right)\end{array}$ & $\begin{array}{c}\text { ISO Bright. } \\
(\%)\end{array}$ & $\begin{array}{c}\text { Tensile Index } \\
(\mathrm{Nm} / \mathrm{g})\end{array}$ \\
\hline Phyllostachys & Cont. & $20,03(0,11)$ & $1164(11,06)$ & $26,91(1,56)$ & $39,04(1,23)$ \\
bambusoides & 3 bar & $7,08(0,07)$ & $889(8,11)$ & $43,59(1,28)$ & $50,72(2,27)$ \\
$\left(50 \pm 5 \mathrm{SR}^{\circ}\right)$ & 5 bar & $6,71(0,09)$ & $882(7,99)$ & $53,29(1,45)$ & $46,34(2)$ \\
& 7 bar & $6,25(0,05)$ & $750(8,54)$ & $53,82(1,85)$ & $41,13(2)$ \\
Populus & Cont. & $29,14(0,08)$ & $1188(10,32)$ & $35,49(1,69)$ & $36,31(5,03)$ \\
euramericana & 3 bar & $14,30(0,06)$ & $1043(11,45)$ & $48,56(2,50)$ & $53,80(2,26)$ \\
$\left(50 \pm 5 \mathrm{SR}^{\circ}\right)$ & 5 bar & $11,36(0,10)$ & $838(7,48)$ & $61,10(2,71)$ & $42,89(7,98)$ \\
& 7 bar & $11,08(0,09)$ & $809(7,66)$ & $63,04(1,49)$ & $42,04(6,80)$ \\
\hline
\end{tabular}

In all trials, $\mathrm{NaOH}, \mathrm{MgSO}_{4}$, time, temperature and consistency were constant at $3 \%, 0,5 \%, 60 \mathrm{~min}, 100{ }^{\circ} \mathrm{C}$ and $12 \%$, respectively. Standard deviations are shown in parentheses 
As shown in table 2, the most appropriate oxygen pressure was 5 bars for bamboo and poplar samples. This was because the tensile index of the bleached samples exhibited a very moderate decrease compared to control samples for bamboo and poplar pulps. Moreover, the kappa number and brightness represent the desired value for other bleaching stages without a severe decline in viscosity. Chelation and SPBTH bleaching were performed after oxygen delignification reached 5 bars. Before the SPBTH bleaching process, pulp exposed to oxygen delignification was pretreated with $0,3 \%$ EDTA (ethylenediamine tetraacetic) for 60 min at $80^{\circ} \mathrm{C}$ in heat-proof polystyrene bags to eliminate undesirable heavy metal ions (chelating stage). The pulp consistency was $12 \%$ and $\mathrm{pH}$ ranged from 5 to 7 in this stage. SPBTH bleaching stage was performed as the final stage. The pulp was then placed in heat-proof polystyrene bags. The amount of SPBTH and length of application were set as independent variables, while temperature $\left(70^{\circ} \mathrm{C}\right)$ and concentration $(12 \%)$ were set as constant (Table 3). This is because SPBTH requires high temperatures such as 70 to $80^{\circ} \mathrm{C}$ and medium concentrations for activation (Pesman et al. 2014).

Table 3. SPBTH bleaching conditions for bamboo and poplar.

\begin{tabular}{lc}
\hline SPBTH level $(\%)$ & $2,4,6$ \\
Treatment time (min) & $60,70,80$ \\
$\mathrm{MgSO}_{4}(\%)$ & 0,5 \\
Temperature $\left({ }^{\circ} \mathrm{C}\right)$ & 70 \\
Consistency $(\%)$ & 12 \\
\hline
\end{tabular}

\section{Refining Process}

Each sample of unbleached and bleached bamboo and poplar pulp (30 Schopper Riegler degrees $\left(\mathrm{SR}^{\circ}\right) \pm 5$ ) was beaten using two different beating degrees $\left(55 \mathrm{SR}^{\circ} \pm 5\right.$ and $\left.65 \mathrm{SR}^{\circ} \pm 5\right)$ at 3000 revolutions in the PFI. Beating characteristics were examined using the pulp $\mathrm{SR}^{\circ}$.

\section{Evaluation of the pulp}

Determination of kappa numbers and viscosities of pulp samples was carried out according to standard procedures described in T 236 om-99 and SCAN 15:62, respectively. The determination of viscosity and kappa number were carried out three times for each sample. Handsheets of $60 \mathrm{~g} / \mathrm{m}^{2}$ were formed and their properties were evaluated in accordance with T410 om-98. The handsheets were conditioned in accordance with T402 sp-98. Tensile index of handsheets was measured using the T404 cm-92 method. Brightness was calculated to ISO standard (ISO 2470).

\section{Collecting the experimental data}

The data for the modeling were provided by experiments. The MATLAB Neural Network Toolbox was used for the configuration of the models. The data were randomly divided into three sets, training, validation and testing, for tensile index and brightness. Thirty-eight samples were used for the ANN training process for each of tensile strength and brightness, while the remaining 16 samples were equally divided for validation and testing processes. The data sets used in the models are given in Table 4. The predicted values of the experimental samples for tensile index and brightness, and their percentage errors, are shown in Table 6 . 


\section{Performance criteria}

The mean absolute error (MAE), the mean absolute percentage error (MAPE), the root mean square error (RMSE), and correlation coefficient $\left(\mathrm{R}^{2}\right)$ were used in evaluating the prediction performance of the models. The models yielding the best results for tensile index and brightness were considered as the prediction models. Performance criteria were calculated using Eq. (2), (3), (4) and (5).

$$
\begin{aligned}
\text { MAE } & =\frac{1}{N} \sum_{i=1}^{N}\left|t_{i}-t_{i}\right| \\
\text { MAPE } & =\frac{1}{N}\left(\sum_{i=1}^{N}\left[\left|\frac{t_{i}-t_{i}}{t_{i}}\right|\right]\right) \times 100 \\
\text { RMSE } & =\sqrt{\frac{1}{N} \sum_{i=1}^{N}\left(t_{i}-t_{i}\right)^{2}} \\
R^{2} & =1-\frac{\sum_{i=1}^{N}\left(t_{i}-t_{i}\right)^{2}}{\sum_{i=1}^{N}\left(t_{i}-\bar{t}\right)^{2}}
\end{aligned}
$$

where, $\mathrm{t}_{\mathrm{i}}$ is the measured (experimental) values, $\mathrm{td}_{\mathrm{i}}$ is the $\mathrm{ANN}$ predicted values, $\mathrm{N}$ is the total number of training patterns and $\bar{t}$ is the average of predicted values.

\section{Neural network architectures}

The networks were trained using different network architecture and learning parameters. The models were then tested using the test data sets of tensile index and brightness for evaluating the performance of the networks. The models giving the most accurate results were thus targeted. The models producing the values closest to the measured values were therefore used for prediction. Wood species, SPBTH ratio, time and beating degree were considered as input variables. Tensile index and brightness were used as output variables in the models. The number of hidden layer neurons was five in both models, the number being determined by trying various networks. The hyperbolic tangent in the hidden layer and the linear transfer function in the output layer were used as the activation functions. The Levenberg-Marquardt back propagation algorithm was chosen as the training algorithm. 


\section{RESULTS AND DISCUSSION}

Table 4 shows the measured values and standard deviations of tensile index and brightness.

Table 4. Measured (experimental) values and standard deviations of tensile index and brightness.

\begin{tabular}{|c|c|c|c|c|c|c|c|c|c|c|c|c|c|c|}
\hline \multirow{3}{*}{$\begin{array}{l}\text { Wood } \\
\text { species }\end{array}$} & \multirow{3}{*}{\multicolumn{2}{|c|}{$\underset{\text { (min.) }}{\text { Time }} \mathrm{SR}^{\mathrm{o}}$}} & \multicolumn{6}{|c|}{ Tensile index $(\mathrm{Nm} / \mathrm{g})$} & \multicolumn{6}{|c|}{ ISO Brightness (\%) } \\
\hline & & & \multicolumn{2}{|c|}{$2 \%$} & \multicolumn{2}{|c|}{$4 \%$} & \multicolumn{2}{|c|}{$6 \%$} & \multicolumn{2}{|c|}{$2 \%$} & \multicolumn{2}{|c|}{$4 \%$} & \multicolumn{2}{|c|}{$6 \%$} \\
\hline & & & $\mathrm{m}$ & SD & $\mathrm{m}$ & SD & $\mathrm{m}$ & SD & $\mathrm{m}$ & $\mathrm{SD}$ & $\mathrm{m}$ & $\mathrm{SD}$ & $\mathrm{m}$ & SD \\
\hline \multirow{9}{*}{$\begin{array}{l}\text { Populus } \\
\text { euramericana }\end{array}$} & \multirow{3}{*}{60} & 30 & 21,88 & 2,45 & 20,42 & 3,52 & 18,99 & 6,33 & 68,91 & 0,51 & 70,09 & 0,44 & 72,67 & 0,66 \\
\hline & & 55 & 48,29 & 6,46 & 51,80 & 4,33 & 44,81 & 4,51 & 66,63 & 0,51 & 68,80 & 0,12 & 70,26 & 0,39 \\
\hline & & 65 & 62,47 & 5,25 & 54,83 & 5,34 & 52,59 & 3,07 & 65,37 & 0,32 & 68,17 & 0,18 & 69,37 & 0,40 \\
\hline & \multirow{3}{*}{70} & 30 & 24,39 & 1,23 & 23,44 & 2,76 & 23,24 & 2 & 69,14 & 0,62 & 70,82 & 1,20 & 72,81 & 0,55 \\
\hline & & 55 & 58,17 & 3,18 & 47,60 & 3,68 & 46,56 & 3,15 & 67,47 & 0,21 & 69,30 & 0,21 & 70,88 & 0,34 \\
\hline & & 65 & 63,64 & 6,90 & 52,80 & 4,08 & 52,42 & 4,53 & 66,92 & 0,45 & 68,66 & 0,32 & 70,63 & 0,21 \\
\hline & \multirow{4}{*}{80} & 30 & 21,05 & 3,32 & 21,37 & 2,08 & 25,31 & 1,75 & 69,30 & 0,25 & 71,57 & 0,19 & 73,08 & 0,23 \\
\hline & & 55 & 42,41 & 3,90 & 42,20 & 4,15 & 50,98 & 4,77 & 67,42 & 0,23 & 70,32 & 0,17 & 71,10 & 0,17 \\
\hline & & 65 & 57,51 & 4,92 & 49,56 & 2,33 & 54,21 & 5,67 & 65,97 & 0,82 & 68,86 & 1,19 & 70,74 & 0,19 \\
\hline \multirow{9}{*}{$\begin{array}{l}\text { Phyllostachys } \\
\text { bambusoides }\end{array}$} & & 30 & 9,88 & 1,50 & 12,83 & 2,42 & 12,72 & 1,55 & 59,83 & 0,59 & 61,37 & 0,61 & 63,13 & 0,23 \\
\hline & \multirow[t]{2}{*}{60} & 55 & 42,44 & 1,87 & 51,51 & 1,80 & 51,01 & 5,12 & 59,79 & 0,40 & 61,60 & 0,60 & 62,62 & 0,31 \\
\hline & & 65 & 45,70 & 8,57 & 58,66 & 2,80 & 54,63 & 4,66 & 60,66 & 0,47 & 61,41 & 0,55 & 62,28 & 0,28 \\
\hline & \multirow{3}{*}{70} & 30 & 12,65 & 2,06 & 12,33 & 1,39 & 9,88 & 1,77 & 61,11 & 0,18 & 64,70 & 0,15 & 62,48 & 0,22 \\
\hline & & 55 & 53,45 & 3,24 & 52,38 & 2,09 & 51,50 & 3,87 & 61,38 & 0,32 & 63,98 & 0,32 & 62,26 & 0,38 \\
\hline & & 65 & 56,34 & 5,54 & 56,47 & 3,96 & 62,09 & 3,74 & 61,86 & 1 & 64,54 & 0,48 & 62,36 & 0,62 \\
\hline & \multirow{3}{*}{80} & 30 & 13,97 & 1,90 & 13,01 & 2,18 & 13,38 & 1,04 & 60,75 & 0,54 & 64,16 & 0,28 & 64,15 & 0,21 \\
\hline & & 55 & 54,12 & 3,16 & 57,83 & 2,64 & 52,95 & 2,94 & 61 & 0,26 & 64,46 & 0,23 & 64,30 & 0,16 \\
\hline & & 65 & 58,76 & 4,65 & 59,78 & 3,12 & 62,35 & 2,40 & 60,98 & 0,42 & 64,17 & 0,28 & 64,12 & 0,25 \\
\hline
\end{tabular}

$\mathrm{m}$ and SD denote measured (experimental) value and standard deviation, respectively

Note; bold values: validation data, bold italic values: testing data, the other values: training data

$2 \%, 4 \%$ and $6 \%$ indicate SPBTH ratio $(\%)$

The tensile index and brightness results of the bamboo and poplar pulp, to which SPBTH bleaching was applied after 5 bar oxygen delignification, are presented in table 4 . This shows that increasing the SPBTH ratio slightly increased the pulp brightness for bamboo and poplar papers. Sodium perborate is a solid form of hydrogen peroxide and sodium metaborate (Peşman et al. 2014). The bleaching mechanism of sodium perborate is therefore similar to that of hydrogen peroxide. Hydroperoxide anion ( $\left.\mathrm{HOO}^{-}\right)$is an active species and is responsible for the bleaching action of hydrogen peroxide under alkaline conditions, but sodium perborate does not require extra alkaline for bleaching activity (Parthasarathy et al. 1990). The brightness values of bamboo pulp were lower than poplar pulp in this study, probably due to the different lignin content in bamboo and poplar samples. Bamboo has been reported to have generally higher lignin content than poplar (Akgül et al. 2002, Okan et al. 2013). Examination of the effect of SPBTH ratio on tensile index of bleached bamboo and poplar handsheet revealed an increase in the SPBTH charge to above $2 \%$, showing a generally positive effect for bamboo pulp but a negative effect on tensile index for poplar. However, the tensile index decreased with a $6 \%$ SPBTH level for poplar and bamboo pulps. This may be due to the decomposition of the organic structure in pulp because holocellulose is the main component contributing to the strength of paper. Hydroxyl radicals, which are generated by decomposition of hydrogen peroxide, are capable of attacking practically all types of organic structure, including those containing hydroxyl and ether linkages, resulting in strength loss in fibers (Parthasarathy et al. 1990). Celik et al. (2008), reported that the increased sodium perborate monohydrate ratio for kraft pulp increased the brightness. Varranes et al. (1996), observed a positive effect of sodium perborate monohydrate on pulp brightness and tensile index. Similar results were reported by Sain et al. (1997). 
Bleaching time is one of the most important parameters of pulp and paper bleaching. In this study, an increase in the bleaching time generally exhibited a positive effect on brightness for bamboo and poplar pulps. It is assumed that the longer the SPBTH is allowed to react, the more it is beneficially consumed. Table 4 shows that the tensile index is generally increased by increasing the bleaching time. For bamboo, bleaching time has a generally positively effect on tensile index. This may be attributed to an increase in the rate of lignin removal having a positive impact on the paper's physical properties (Okan et al. 2013). Li et al. (2011), applied unbleached eucalyptus kraft pulp on treatment different bleaching time with hydrogen peroxide after xylan adsorption. The tensile index dropped first and then rose again after bleaching for 60 to $120 \mathrm{~min}$. In order to understand this phenomenon, the chemical and physical properties of pulp in the bleaching process must be analyzed (Li et al. 2011).

Beating degree affected pulp strength strongly and brightness slightly. Strength of paper increases with pulp beating or refining since it relies on fiber-to-fiber bonding (Kamthai and Puthson 2005). Tensile index increased by raising the $\mathrm{SR}^{\circ}$ degree, but brightness decreased slightly with increasing beating. This may be due to the impurities caused by metal cups and interference during prolonged beating (Ibrahem et al. 1989). Fišerová et al. (2010), found that the tensile index of unbleached and bleached softwood and hardwood kraft pulps increased by raising the beating degree.

Analysis of variance (ANOVA) was performed to determine the effects of the wood species, SPBTH ratio, time, and beating degree parameters on tensile index and brightness. The results are given in table 5 .

Table 5. Results of the Duncan test for the various parameters for tensile index and brightness

\begin{tabular}{|c|c|c|c|c|c|}
\hline \multirow{2}{*}{\multicolumn{2}{|c|}{ Treatment conditions }} & \multicolumn{2}{|c|}{ Tensile index $(\mathrm{Nm} / \mathrm{g})$} & \multicolumn{2}{|c|}{ ISO Brightness (\%) } \\
\hline & & Average & $\mathrm{HG}$ & Average & $\mathrm{HG}$ \\
\hline \multirow{2}{*}{$\begin{array}{l}\text { Wood } \\
\text { species }\end{array}$} & $\begin{array}{c}\text { Populus } \\
\text { euramericana }\end{array}$ & 41,96 & A & 69,45 & A \\
\hline & $\begin{array}{l}\text { Phyllostachys } \\
\text { bambusoides }\end{array}$ & 40,47 & B & 62,42 & B \\
\hline \multirow{3}{*}{$\begin{array}{c}\text { SPBTH ratio } \\
(\%)\end{array}$} & 2 & 41,51 & A & 64,14 & A \\
\hline & 4 & 41,05 & $\mathrm{~A}$ & 66,50 & B \\
\hline & 6 & 41,09 & A & 67,18 & $\mathrm{C}$ \\
\hline \multirow{3}{*}{ Time (min) } & 60 & 39,75 & A & 65,16 & A \\
\hline & 70 & 41,71 & B & 66,18 & B \\
\hline & 80 & 42,19 & B & 66,47 & $\mathrm{C}$ \\
\hline \multirow{3}{*}{$\begin{array}{c}\text { Beating } \\
\text { degree (SR })\end{array}$} & 30 & 17,26 & A & 66,67 & A \\
\hline & 55 & 49,99 & B & 65,75 & B \\
\hline & 65 & 56,38 & $\mathrm{C}$ & 65,39 & $\mathrm{C}$ \\
\hline
\end{tabular}

According to the results of the analysis of variance, except for the effect of SPBTH ratio on tensile index, the effect of the parameters on tensile index and brightness was statistically significant with a $5 \%$ error margin. The Duncan test was then applied to detect difference or similarity in the parameters on tensile index and brightness between the groups. The differences and similarities determined are shown in the columns of homogenous groups (HG). The same letters in the columns of homogenous groups indicate that there are no differences between the groups in terms of the effect on tensile index or brightness, while different letters show that there are differences between the groups in terms of the effect on tensile index or brightness. For example, the effect of SPBTH ratio $(2 \%, 4 \%$ and $6 \%)$ on tensile index is not statically significant (due to being the same HG), while its effect on brightness is statically significant (due to being a different HG).

The predicted values and percentage errors of the experimental samples of tensile index and brightness are given in table 6. 
Table 6. Predicted values and percentage errors of tensile index and brightness.

\begin{tabular}{|c|c|c|c|c|c|c|c|c|c|c|c|c|c|c|}
\hline \multirow{3}{*}{$\begin{array}{l}\text { Wood } \\
\text { species }\end{array}$} & \multirow{3}{*}{$\begin{array}{l}\text { Time } \\
\text { (min.) }\end{array}$} & \multirow{3}{*}{$\mathrm{SR}^{\mathrm{o}}$} & \multicolumn{6}{|c|}{ Tensile index $(\mathrm{Nm} / \mathrm{g})$} & \multicolumn{6}{|c|}{ ISO Brightness (\%) } \\
\hline & & & \multicolumn{2}{|c|}{$2 \%$} & \multicolumn{2}{|c|}{$4 \%$} & \multicolumn{2}{|c|}{$6 \%$} & \multicolumn{2}{|c|}{$2 \%$} & \multicolumn{2}{|c|}{$4 \%$} & \multicolumn{2}{|c|}{$6 \%$} \\
\hline & & & $\mathrm{p}$ & $\mathrm{e}$ & $\mathrm{p}$ & $\mathrm{e}$ & $\mathrm{p}$ & $\mathrm{e}$ & $\mathrm{p}$ & e & $\mathrm{p}$ & $\mathrm{e}$ & $\mathrm{p}$ & $\mathrm{e}$ \\
\hline \multirow{9}{*}{$\begin{array}{l}\text { Populus } \\
\text { euramericana }\end{array}$} & \multirow{3}{*}{60} & 30 & 23,50 & $-7,39$ & 21,19 & $-3,78$ & 17,04 & 10,27 & 67,62 & 1,58 & 70,10 & $-0,02$ & 72,55 & 0,17 \\
\hline & & 55 & 57,17 & $-18,39$ & 51,90 & $-0,19$ & 46,10 & $-2,88$ & 66,49 & 0,21 & 68,71 & 0,13 & 70,33 & $-0,11$ \\
\hline & & 65 & 62,68 & $-0,34$ & 55,89 & $-1,94$ & 50,16 & 4,61 & 65,55 & $-0,28$ & 68,13 & 0,05 & 69,36 & 0,03 \\
\hline & \multirow{3}{*}{70} & 30 & 22,91 & 6,08 & 19,94 & 14,92 & 20,33 & 12,51 & 69,28 & $-0,20$ & 71,17 & $-0,51$ & 72,71 & 0,13 \\
\hline & & 55 & 54,49 & 6,33 & 50,28 & $-5,63$ & 44,45 & 4,54 & 67,71 & $-0,35$ & 69,49 & $-0,27$ & 71,01 & $-0,18$ \\
\hline & & 65 & 60,40 & 5,10 & 53,74 & $-1,77$ & 49,32 & 5,91 & 66,77 & 0,22 & 68,68 & $-0,02$ & 70,47 & 0,23 \\
\hline & \multirow{4}{*}{80} & 30 & 23,11 & $-9,79$ & 19,08 & 10,70 & 26,05 & $-2,92$ & 69,11 & 0,27 & 71,83 & $-0,36$ & 73,02 & 0,085 \\
\hline & & 55 & 44,29 & $-4,42$ & 41,81 & 0,92 & 50,27 & 1,40 & 67,51 & $-0,14$ & 69,87 & 0,64 & 71,03 & 0,10 \\
\hline & & 65 & 58,04 & $-0,93$ & 51,83 & $-4,56$ & 55,12 & $-1,67$ & 66,46 & $-0,75$ & 69 & $-0,20$ & 70,29 & 0,63 \\
\hline \multirow{9}{*}{$\begin{array}{l}\text { Phyllostachys } \\
\text { bambusoides }\end{array}$} & & 30 & 11,24 & $-13,72$ & 13,93 & $-8,60$ & 13,61 & -7 & 59,94 & $-0,18$ & 61,69 & $-0,51$ & 62,97 & 0,26 \\
\hline & \multirow[t]{2}{*}{60} & 55 & 42,11 & 0,76 & 50,82 & 1,35 & 50,48 & 1,03 & 59,83 & $-0,06$ & 61,71 & $-0,19$ & 62,52 & 0,16 \\
\hline & & 65 & 45,89 & $-0,42$ & 57,34 & 2,24 & 54,71 & $-0,16$ & 60,60 & 0,10 & 62,20 & $-1,28$ & 62,47 & $-0,31$ \\
\hline & \multirow{4}{*}{70} & 30 & 13,80 & $-9,09$ & 12,26 & 0,50 & 11,74 & $-18,91$ & 61,45 & $-0,57$ & 64,37 & 0,51 & 62,54 & $-0,09$ \\
\hline & & 55 & 52,12 & 2,49 & 50,94 & 2,75 & 50,41 & 2,12 & 61,30 & 0,14 & 64,25 & $-0,43$ & 62,25 & 0,02 \\
\hline & & 65 & 55,54 & 1,42 & 58,63 & $-3,82$ & 61,08 & 1,63 & 61,92 & $-0,11$ & 64,82 & $-0,44$ & 62,36 & -0 \\
\hline & & 30 & 12,82 & 8,20 & 11,39 & 12,50 & 10,88 & 18,66 & 61,18 & $-0,71$ & 64,33 & $-0,26$ & 64,27 & $-0,19$ \\
\hline & \multirow[t]{2}{*}{80} & 55 & 58,07 & $-7,30$ & 55,61 & 3,83 & 52,51 & 0,83 & 61,02 & $-0,04$ & 63,90 & 0,86 & 64,11 & 0,29 \\
\hline & & 65 & 58,63 & 0,22 & 60,51 & $-1,22$ & 62,59 & $-0,385$ & 61,61 & $-1,03$ & 64,02 & 0,23 & 64,55 & $-0,67$ \\
\hline
\end{tabular}

$\mathrm{p}$ and e denote predicted values and errors in \%, respectively

Note; bold values: validation data, bold italics values: testing data, the other values: training data

$2 \%, 4 \%$ and $6 \%$ indicate SPBTH ratio (\%)

Comparing predicted values to measured values, in most cases the neural network prediction is very close to the measured values. However, some values are not as close as others. Canakc1 et al. 2012, suggested that this is due to the errors caused by the material, the measurements and process parameters, and can be neglected.

Performance criteria values used to assess the performance of the proposed prediction models are given in Table 7. The prediction values were clearly determined with very low percentage errors. The low level of errors is satisfactory for predicting tensile index and brightness. This also demonstrates that the networks effectively give accurate results.

Table 7. Performance criteria used for predicting tensile index and brightness by ANNs.

\begin{tabular}{|c|c|c|c|c|c|c|}
\hline \multirow{3}{*}{$\begin{array}{l}\text { Performance } \\
\text { criteria }\end{array}$} & \multicolumn{6}{|c|}{ Data sets } \\
\hline & \multicolumn{3}{|c|}{ Tensile index $(\mathrm{Nm} / \mathrm{g})$} & \multicolumn{3}{|c|}{ Brightness (\%) } \\
\hline & Training & Validation & Testing & Training & Validation & Testing \\
\hline MAPE & 3,883 & 7,711 & 8,984 & 0,191 & 0,655 & 0,658 \\
\hline MAE & 1,229 & 1,916 & 2,758 & 0,127 & 0,411 & 0,441 \\
\hline RMSE & 1,556 & 2,046 & 3,757 & 0,158 & 0,460 & 0,570 \\
\hline $\mathrm{R}^{2}$ & 0,992 & 0,998 & 0,980 & 0,998 & 0,998 & 0,985 \\
\hline
\end{tabular}

Regression analysis between the predicted values and the measured values is generally used to assess the validity of the networks and their accuracy. Figures 2 and 3 show the relationship between the measured values and predicted values for training data, validation data, testing data and all data in predicting tensile index and brightness, respectively. 

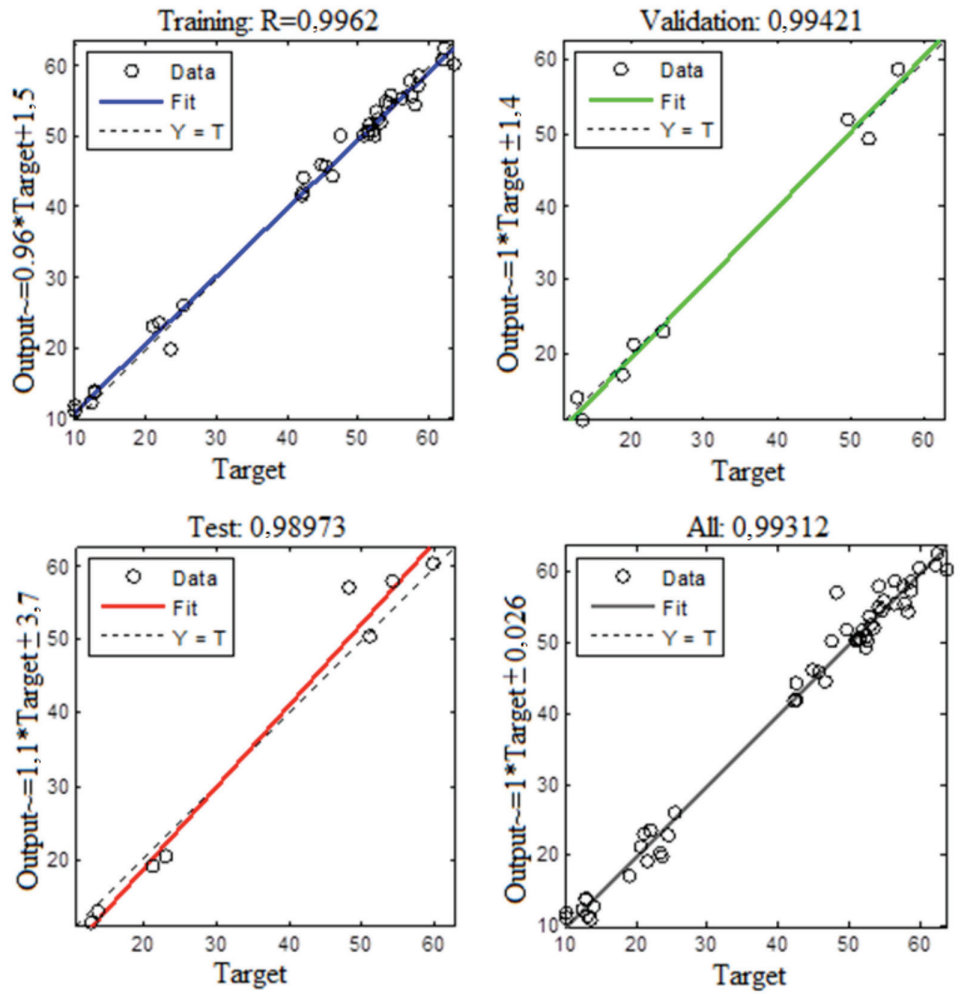

Figure 2. Relationship between measured results and predicted results for tensile index.
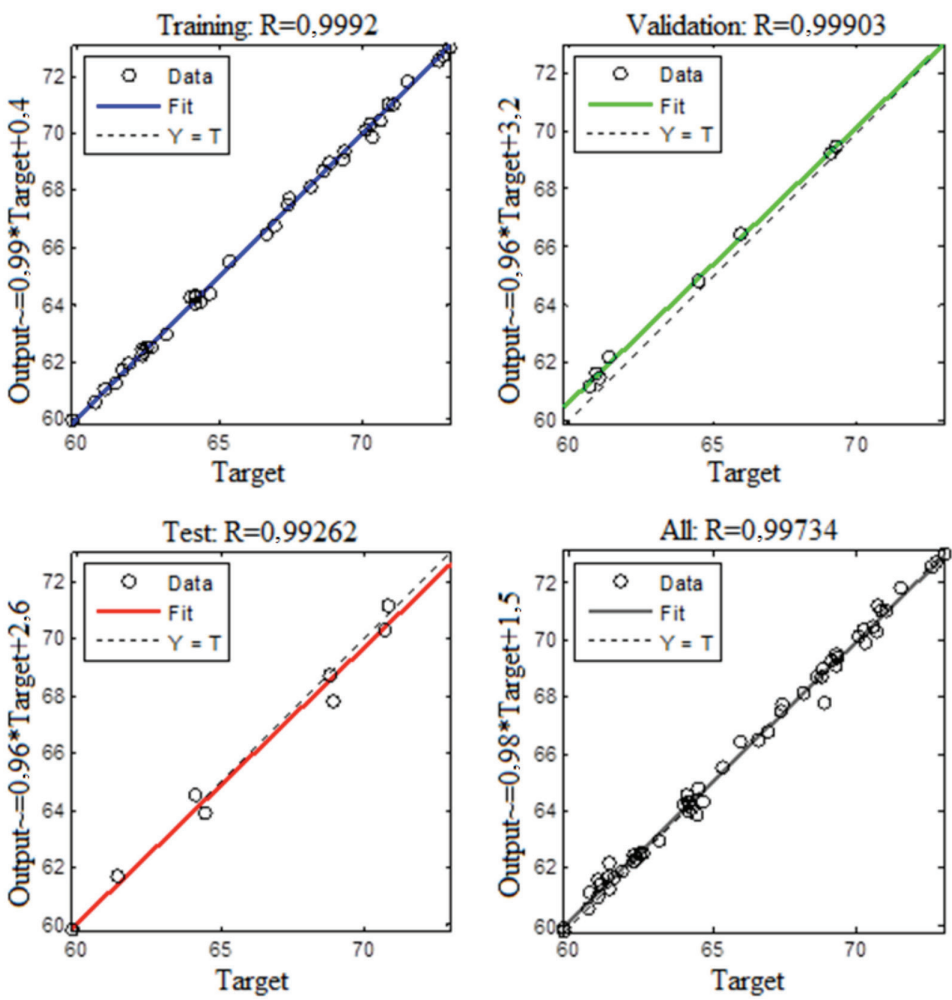

Figure 3. Relationship between measured results and predicted results for brightness. 
In the figures, the predicted values are plotted against the measured values as open circles. The best linear relationship is shown with a dashed line. In addition, the perfect linear relationship between measured values and the predicted values in predicting tensile index and brightness is indicated by a solid line.

The accuracy of the prediction models is proved by the increasing correlation coefficient $\left(\mathrm{R}^{2}\right)$ values. As $\mathrm{R}^{2}$ approaches 1, prediction accuracy increases. Figure 2 shows $\mathrm{R}$ values for training, validation and testing data sets in predicting tensile index of 0,$9962 ; 0,9942$ and 0,9897 ; respectively. Thus, the $\mathrm{R}^{2}$ values are greater than 0,99 for training; 0,98 for validation and equal to 0,98 for testing. According to figure 3 , the $\mathrm{R}$ values for training, validation and testing data sets in predicting brightness are 0,9992; 0,9990 and 0,9926; respectively. The $\mathrm{R}^{2}$ values are therefore greater than 0,99 for training and validation, and 0,98 for testing data set. The results indicate that the networks obtained explain at least $98 \%$ of the experimental data for all data sets. This means that there is a good relationship between the measured results and the prediction results. Zainuddin et al. 2011, obtained an $\mathrm{R}^{2}$ value of $96,47 \%$ in the prediction of tensile index values in the of oil palm frond pulping process using a neural network method. In the present study, the results obtained by the ANN allowed prediction with a higher $\mathrm{R}^{2}$ value compared to that study.

Figure 4, shows the comparison of measured values and predicted values of tensile index and brightness.
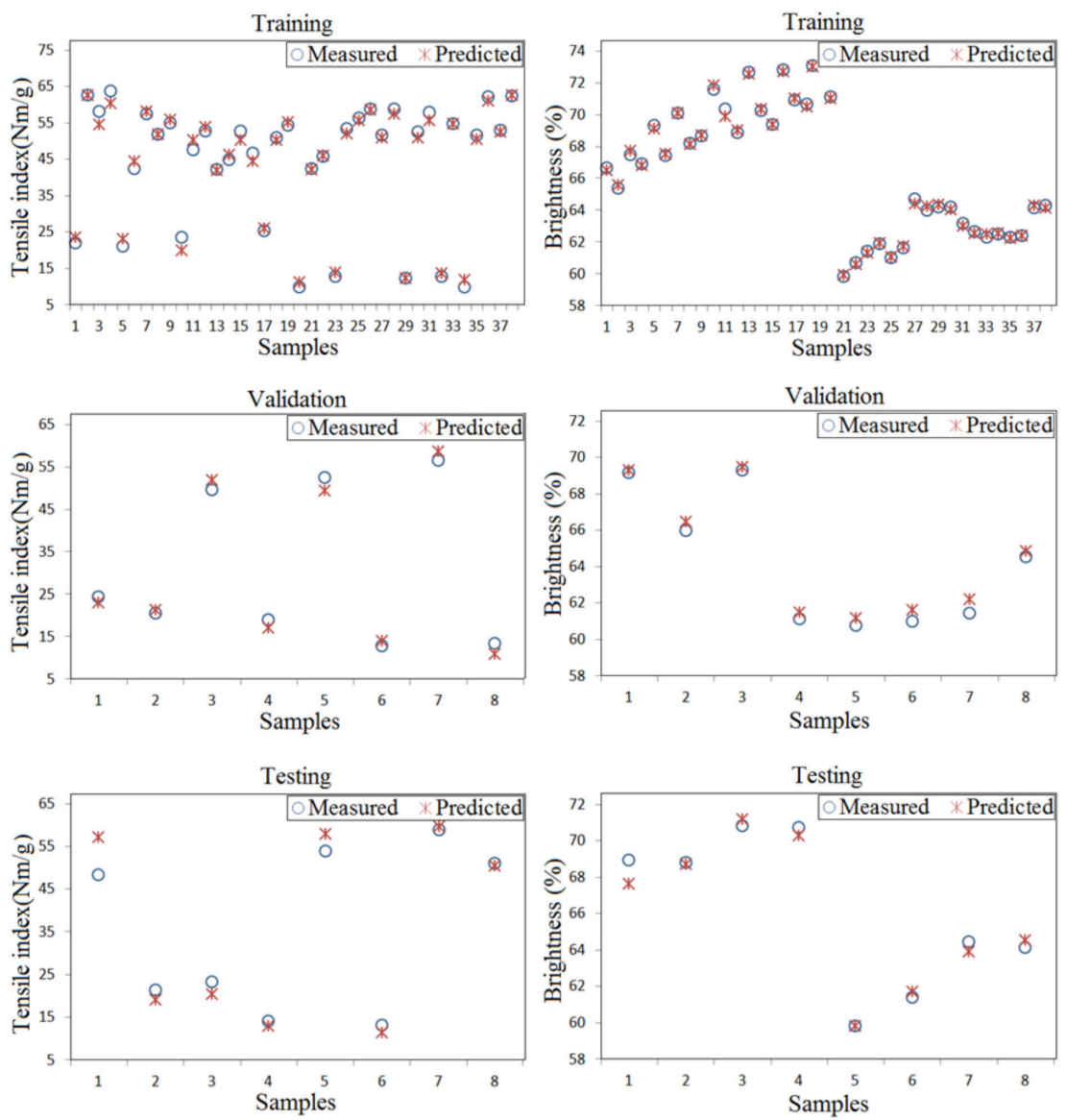

Figure 4. Comparison of the measured and predicted values for the training, validation and testing data sets for tensile index and brightness. 
Analysis of the comparison graphics reveals that the predicted values are very close to the measured values. This further increases the reliability of the designed prediction models. In addition, the ANN approach successfully predicts tensile index and brightness without requiring time consuming and costly comprehensive experimental investigation.

\section{CONCLUSIONS}

Tensile index and brightness values in bleaching pulp were predicted by ANNs using the experimental results.

The experimental results showed that the tensile index was generally positively affected with increasing bleaching time for bamboo, but that the tensile index for poplar first increased and then decreased with bleaching time. Additionally, poplar exhibited a higher tensile index and brightness compared to bamboo. An increased beating degree has a positive effect on tensile index while brightness was adversely affected for both poplar and bamboo. Finally, the SPBTH ratio had only a slight effect on the tensile index, while it increased brightness.

The prediction results are highly satisfactory in terms of explanatory characteristics and validity of the models. The results of the research indicate that the ANN approach can be successfully used to model the effects of wood species, SPBTH ratio, time, and beating degree on tensile index and brightness in the production of pulp without the need for further experimental investigation. This study therefore permits a preliminary decision to be made concerning usability under conditions in which the tensile index and brightness properties of paper is important. The proposed models also save time, reduce the consumption of experimental materials and lower design costs. 


\section{REFERENCES}

Akgül, M.; Kırcı, H. 2002. Dissolving pulp obtaining possibilities by organosolv pulp from poplar wood. KSU J. Science and Engineering 5(1):72-85.

Atchison, J.E. 1998. Update on global use of non-wood plant fibers and some prospects of their greater use in United States, Proceeding of the 1998 TAPPI North American Non-Wood Fiber Symposium, 17-18 Febuary, Atlanta, GA, USA, 13-42.

Atik, C.; Imamoglu, S.; Bermek, H. 2006. Impact of xylanase pre-treatment on peroxide bleaching stage of biokraft pulp. International Biodeterioration \& Biodegradation 58: 22-26.

Baily, D.; Thompson D.M. 1990. Developing neural network applications. AI Expert (September), 33-41.

Canakci, A.; Ozsahin, S.; Varol, T. 2012. Modeling the influence of a process control agent on the properties of metal matrix composite powders using artificial neural networks. Powder Technology 228: 26-35.

Celik, A.G.; Saka, M.; Celen, E.M.; Cakal, O.G. 2008. Fresh paper pulp bleached with sodium perborate monohydrate by factor. II National Boron Workshop, 311-331. Turkey.

Ceylan, I. 2008. Determination of drying characteristics of timber by using artificial neural networks and mathematical models. Drying Technology 26(12):1469-1476.

Esteban, L.G.; Fernandez, F.G.; de-Palacios, P. 2011. Prediction of plywood bonding quality using an artificial neural network. Holzforschung 65:209-214.

Ferrer, A.; Rosal, A.; Valls, C.; Roncero, B.; Rodriguez, A. 2011. Modeling hydrogen peroxide bleaching of soda pulp from oil-palm empty fruit bunches. Bioresources 6(2):1298-1307.

Fišerová, M.; Gıgac, J.; Balberčák, J.; 2010. Relatıonshıp between fibre characterıstıcs and tensıle strength of hardwood and softwood kraft pulps. Cellulose Chemistry and Technology 44(7-8):249-253.

Francis, R. C.; Hanna, R.B.; Shin, S.J.; Brown, A.F.; Riemenschneider, D.E. 2006. Papermaking characteristics of three Populus clones grown in the north-central United States. Biomass and Bioenergy 30:803-808.

Hamzacebi, C.; Akay, D.; Kutay, F. 2009. Comparison of direct and iterative artificial neural network forecast approaches in multi-periodic time series forecasting. Expert Systems with Applications 36:3839-3844.

Hamzacebi, C. 2011. Artificial neural networks. 1. Edition, Ekin publishing, Bursa.

Ibrahem, A.A.; Yousef, M.A.; El-Meadawy, S.A. 1989. Effect of beating on fibre crystallinity and physical properties of paper shets. Production Engineering 2(4):295-298.

Jimenez, L.; Navarro E.; Ferrer, J.L.; Lopez, F.; Ariza, J. 1999. Biobleaching of cellulose pulp from wheat straw with enzymes and hydrogen peroxide. Journal of procbiotics 35:149-157.

Kamthai, S.; Puthson, P. 2005. Effect of beating revolution on sweet bamboo (Dendrocalamus asper backer) Kraft pulp properties. CMU journal 4(2):137-147.

Lippmann, R.P. 1987. An introduction to computing with neural nets. IEEE ASSP Magazine, April, 4-22. 
Li, L.; Lee, S.; Lee, H.L.; Youn, H.Y. 2011. Hydrogen peroxide bleaching of hardwood kraft pulp with adsorbed birch xylan and its effect on paper properties. Bioresources 6(1):721-736.

Lopez, F.; Diaz, M.J.; Eugenio, M.E.; Jimenez, L. 2002. Hydrogen peroxide and sodium perborate bleaching of pulp from olive tree residues. Engineering in Life Sciences 2(7):201-208.

Masters, T. 1993. Practical neural network recipes in $C++$. Academic Press, New York.

Moldes, D.; Diaz, M.; Tzanov, T.; Vidal, T. 2008. Comparative study of the efficiency of synthetic and natural mediators in laccase-assisted bleaching of Eucalyptus kraft pulp. Bioresource Technology 99:7959-7965.

Nykvist, M. 2006. Improved brightness stability of softwood pulp. Department of chemical engineering, Master Thesis, Lund University, Sevenska.

Okan, O.T.; Deniz, I.; Yildirim, I. 2013. Bleaching of bamboo (Phyllostachys bambusoides) Kraft-AQ Pulp with sodium perborate tetrahydrate (SPBTH) after oxygen delignification. Bioresources 8(1):1332-1344.

Parthasarathy, V.R.; Klein, R.; Sundaram, V.S.M.; Jammel, H.; Gratzl, J.S. 1990. Hydrogen peroxidereinforced oxygen delignification of Southern pine Kraft Pulp and Short sequence bleaching. Tappi Journal 73(7):177-185.

Pesman, E.; Imamoglu, S.; Kalyoncu, E.; Kırcı, H. 2014. The effect of sodium percarbonate and perborate usage on pulping and flotation deinking instead of hydrogen peroxide. Bioresources 9(1):523-536.

Rahmati, H.; Ebrahimi, P.; Sedghi, M. 2010. Effect of cooking conditions and oxygen- delignification on Bambusa tulda kraft pulping. Indian Journal of Chemical Technology.17:74-77.

Rajesh, R.; Ray, A.K. 2006. Artifical neural network for solving paper industry problems: A review. Journal of Scientific and Industrial Research 65:565-573.

Sain, M.M.; Daneault, C.; Parenteau, M.İ. 1997. Bleach activation of thermomechanical pulp. The Canadian Journal of Chemical Engineering 75:62-69.

Tiryaki, S.; Hamzacebi, C. 2014. Predicting modulus of rupture (MOR) and modulus of elasticity (MOE) of heat treated woods by artificial neural networks. Measurement 49: 266-274.

Varennes, S.; Daneault, C.; Parenteau, M. 1996. Bleaching of thermomechanical pulp with sodium perborate. Tappi Journal 79(3):245-250.

Xu, C.; Qin, M.; Fu, Y.; Liu, N.; Hemming, J.; Bjarne, H.; Willför, S. 2010. Lipophilic extractives in Poulus x euramericana Guariento stemwood and bark. Journal of Wood Chemistry and Technology. 30:105-117.

Zainuddin, Z.; Daud, W.R.W.; Pauline, O.; Shafie, A. 2011. Wavelet neural networks applied to pulping of oil palm fronds. Bioresource Technology 102:10978-10986. 